\title{
A note on a Tarski type fixed-point theorem
}

\author{
Roberto Ghiselli Ricci ${ }^{1}$
}

Accepted: 24 January 2021 / Published online: 11 February 2021

(c) The Author(s) 2021, corrected publication 2021

\begin{abstract}
In this paper we propose a basic fixed-point theorem for correspondences inspired by Tarski's intersection point theorem. This result furnishes an efficient tool to prove the existence of pure strategy Nash equilibria for two player games with possibly discontinuous payoffs functions defined on compact real intervals.
\end{abstract}

Keywords Fixed-point Theorem · Existence of Nash equilibria · Tarski's Theorem

\section{Introduction}

A corollary of a theorem of Tarski, called sometimes an intersection point theorem to distinguish it from the more familiar Tarski's fixed-point theorem, contained in the same article (see Tarski 1955), states that a function $f: X \rightarrow X$ which is quasiincreasing (no downward jumps) has a fixed-point, assuming that $X$ is a complete and densely ordered chain. In this note, we prove an analogous result replacing the function $f$ with a correspondence $R$ under the hypothesis that $X$ is a compact real interval and we apply it to establish existence of pure strategy Nash equilibria in a particular family of two player games with possibly discontinuous payoffs functions. A conclusive example shows the usefulness of this application.

\section{A Tarski type fixed-point theorem for correspondences}

Throughout the paper, we will exclusively reserve the letters $X$ and $Y$ for two nonempty compact real intervals. Let $R: X \rightsquigarrow Y$ be a correspondence: we say that $R$ is strict if $R(x)$ is a nonempty subset of $Y$ for all $x \in X$, and closed-valued if $R(x)$ is a closed subset of $Y$ for all $x \in X$. When $Y=X$, we refer to $R$ as a correspondence $o n X$.

Assume that $R: X \rightsquigarrow Y$ is a strict, closed-valued correspondence and denote by $m_{R}: X \rightarrow Y$ and $M_{R}: X \rightarrow Y$ the mappings given by $m_{R}(x)=\min R(x)$

\footnotetext{
Roberto Ghiselli Ricci

ghsrrt@unife.it

1 Department of Economics and Management, University of Ferrara, Ferrara, Italy
} 
and $M_{R}(x)=\max R(x)$ : note that these two functions are well-defined due to the fact that $R$ is closed-valued. Further, denote by $s_{R}: X \rightarrow Y$ the mapping such that $s_{R}(x)$ is the supremum of the maximal connected subset of $R(x)$, also called component, containing $m_{R}(x)$ : note that this function is well defined because of the properties of the components (see, for instance, Kelley 2017). Trivially, we have that $m_{R}(x) \leq s_{R}(x) \leq M_{R}(x)$ and $\left[m_{R}(x), s_{R}(x)\right] \subseteq R(x)$ for any $x \in X$.

Remark 1 Note that one can always associate with each strict and closed-valued correspondence $R$ on $X$ the correspondence $R^{*}: X \rightsquigarrow X$ defined by $R^{*}(x)=$ $\left[m_{R}(x), s_{R}(x)\right]$. Obviously, $R^{*}$ is strict and closed-valued: moreover, $R^{*}$ is convexvalued, i.e. $R^{*}(x)$ is convex for all $x \in X$.

Given any correspondence $R$ on $X$, we say that $x_{0} \in X$ is a fixed-point for $R$ if $x_{0} \in R\left(x_{0}\right)$ and denote with $\mathcal{F}(R)$ the (possibly empty) subset of $X$ of fixed-points of $R$.

Now, we are ready to present the main result of this paper, which is deeply inspired by Tarski's intersection point theorem (see Theorem 3 in Tarski (1955)).

Theorem 1 Let $R$ be a strict, closed-valued correspondence on X. Assume that

$$
\limsup _{x \uparrow x_{0}} s_{R}(x) \leq s_{R}\left(x_{0}\right) \text { for all } x_{0} \in X
$$

and

$$
\underset{x \downarrow x_{0}}{\lim \sup _{R}} m_{R}(x) \geq m_{R}\left(x_{0}\right) \text { for all } x_{0} \in X \text {. }
$$

Then $\mathcal{F}(R)$ is not empty.

Proof Suppose $a b$ absurdo that $\mathcal{F}(R)$ is empty: it is not difficult to see that this is equivalent to saying that $X$ may be partitioned into two disjoint, non-void subsets $S$ and $T$, where $S=\left\{x \in X: x<m_{R}(x)\right\}$ and $T=\left\{x \in X: x>s_{R}(x)\right\}$ (note that $S$ and $T$ are not empty, because at least $a \in S$ and $b \in T$, where $a:=\min X$ and $b:=\max X)$. Let $u=\sup S$ : we assert that

$$
s_{R}(u)>u \text {. }
$$

The above inequality is trivial when $u=a$, so let us consider the case $u>a$. By definition of $u$, there exists a sequence $\left\{x_{n}\right\} \subset S$ such that $x_{n} \uparrow u$ as $n \rightarrow \infty$. Since $x_{n} \in S$, we have that $m_{R}\left(x_{n}\right)>x_{n}$ and thus, a fortiori, $s_{R}\left(x_{n}\right)>x_{n}$ : as a consequence, we claim that $\lim \sup s_{R}(x) \geq u$. Reasoning by contradiction, if $\lim \sup s_{R}(x)=l<$ $x \uparrow u$

$u$, let $\epsilon=h / 2$, where $h=u-l$ : by assumption, there exists a sufficiently small $\delta>0$ such that for any $x \in\left[u-\delta, u\left[\right.\right.$ there holds $\sup s_{R}(x) \leq l+\epsilon$. At the same time, there exists a sufficiently large $\bar{n} \in \mathbb{N}$ such that $x_{\bar{n}} \geq \max \{u-\delta, u-h / 2\}$. Thus, we find $u-h / 2 \leq x_{\bar{n}}<s_{R}\left(x_{\bar{n}}\right) \leq l+\epsilon=u-h / 2$ and the contradiction shows the claim. Now, it is easy to see that the assertion follows from the combination of 
the claim with Eq. (1). Remark that Eq. (3) implies $u<b$, hence $] u, b]$ is a subset of $T$. Consequently, fixing any $x \in] u, b]$, we have that $s_{R}(x)<x$ and thus, a fortiori, $m_{R}(x)<x$ : passing to the limit as $x \rightarrow u$ gives $\lim \sup m_{R}(x) \leq u$, which, combined with Eq. (2), leads to

$$
x \downarrow u
$$

$$
m_{R}(u)<u \text {. }
$$

If we consider both Eqs. (3) and (4), we obtain $u \notin S \cup T=X$, which is a contradiction, so concluding the proof.

Remark 2 In Amir and De Castro (2017), Amir and De Castro provide the real-valued version of Tarski's intersection point theorem for a pair of mappings $f$ and $g$ quasiincreasing and quasi-decreasing, respectively. When $g$ is the identity function, the same result may be read as a fixed-point theorem concerning quasi-increasing maps (see also Milgrom and Roberts 1994), whose set-valued version is just given by Theorem 1 , with the considerable advantage of a simpler proof.

Remark 3 Note that if we apply Theorem 1 to a mapping $f: X \rightarrow X$ and define $R(x)=\{f(x)\}$, then $R$ is obviously a strict and closed-valued correspondence. Moreover, the two assumptions given by Eqs. (1) and (2) are equivalent to the following double inequality

$$
\limsup _{x \uparrow x_{0}} f(x) \leq f\left(x_{0}\right) \leq \limsup _{x \downarrow x_{0}} f(x) \text { for all } x_{0} \in X
$$

Interestingly, as reported by Tarski in Tarski (1955) (see footnote 4), A.P. Morse remarked that, with regard to functions defined on a real interval, Eq. (5) (or else by replacing lim sup with lim inf on both the sides) seems to be the most general assumption able to ensure the existence of fixed-points. To corroborate this statement, recall that, by the previous remark, a quasi-increasing function $f: X \rightarrow X$ admits fixed-points: according to the definition proposed by Amir and De Castro in Amir and De Castro (2017), $f$ is quasi-increasing if it satisfies

$$
\underset{x \uparrow x_{0}}{\lim \sup } f(x) \leq f\left(x_{0}\right) \leq \liminf _{x \downarrow x_{0}} f(x) \text { for all } x_{0} \in X
$$

However, it is immediate to see that every quasi-increasing function fulfills Eq. (5), but not vice versa, as shown by the following example, where $X=[0,1]$ :

$$
f(x)= \begin{cases}\sin ^{2}(1 / x), & \text { if } x \neq 0 \\ 1 / 2, & \text { otherwise }\end{cases}
$$

Remark 4 Observe that, de facto, Theorem 1 establishes the existence of a fixed-point for the correspondence $R^{*}$ associated with $R$ (see Remark 1). The question then arises as to whether Theorem 1 may be derived by applying Kakutani's fixed-point theorem (see Kakutani 1941) to the correspondence $R^{*}$. Well, this question must be answered 
in the negative, because Kakutani's theorem requires a hypothesis not contemplated by Theorem 1, that is the graph $\Gamma=\left\{(x, y) \in X \times X: y \in R^{*}(x)\right\}$ of $R^{*}$ is closed in $X \times X$. Consider, for instance, the correspondence $R:[0,1] \rightsquigarrow[0,1]$ defined as $R(x)=\left\{\sin ^{2}(1 / x), 1\right\}$ for $x>0$, and $R(0)=\{1 / 2\}$. Trivially, $R^{*}$ reduces exactly to the function $f$ introduced in the previous remark. As already noticed, this case is covered by Theorem 1: instead, Kakutani's theorem cannot be applied, because it is clear that the graph of $f$ is not closed.

\section{Pure strategy Nash equilibria in two-player games}

In this section, we will apply Theorem 1 in order to obtain a result about the existence of pure-strategy Nash equilibria in two-player games where the action spaces are compact real intervals.

In the sequel, we will exclusively reserve the letter $\Gamma$ for a two-player strategic game of the kind $(X, Y, f, g)$, where $X$ and $Y$ are the action spaces of the first and the second player, respectively, and $f, g: X \times Y \rightarrow \mathbb{R}$ are the payoff functions of the first and the second player, respectively.

Let $R_{f}: Y \rightsquigarrow X$ and $R_{g}: X \rightsquigarrow Y$ be the best reply correspondences of the first and the second player, respectively, which are defined as $R_{f}(y)=\arg \max _{x \in X} f(x, y)$ and $R_{g}(x)=\arg \max _{y \in Y} g(x, y)$. Let us denote by $R_{f, g}$ the composition product on $X$, or simply the product (see Aubin and Frankowska 2009), of $R_{f}$ and $R_{g}$ which is given by

$$
R_{f, g}(x)=\bigcup_{y \in R_{g}(x)} R_{f}(y) .
$$

Remark 5 Note that, fixing any $x_{0}, z_{0} \in X, z_{0} \in R_{f, g}\left(x_{0}\right)$ if, and only if, there exists a $y_{0} \in Y$ such that

$$
\left\{\begin{array}{l}
f\left(z_{0}, y_{0}\right) \geq f\left(t, y_{0}\right) \text { for all } t \in X \\
g\left(x_{0}, y_{0}\right) \geq g\left(x_{0}, s\right) \text { for all } s \in Y
\end{array}\right.
$$

We say that the strategy profile $\left(x_{0}, y_{0}\right) \in X \times Y$ is a pure strategy Nash equilibrium for the game $\Gamma$ if

$$
\left\{\begin{array}{l}
f\left(x_{0}, y_{0}\right) \geq f\left(t, y_{0}\right) \text { for all } t \in X \\
g\left(x_{0}, y_{0}\right) \geq g\left(x_{0}, s\right) \text { for all } s \in Y
\end{array}\right.
$$

Denote by $E(\Gamma)$ the set of pure-strategy Nash equilibria for the game $\Gamma$.

Corollary $1 E(\Gamma)$ is empty if, and only if, $\mathcal{F}\left(R_{f, g}\right)$ is empty.

Proof Straightforward consequence of Eqs. (6) and (7). 
Recall that, given any mapping $h: X \rightarrow \mathbb{R}$, the upper Baire function of $h$, denoted by $\bar{h}: X \rightarrow \mathbb{R}$, is defined as $\bar{h}(x)=\lim \sup h(z)$.

Given any game $\Gamma$, for the sake of convenience, denote by $f_{t}$ the real mapping on $Y$ defined as $f_{t}(y)=f(t, y)$ for each fixed $t \in X$ and, analogously, denote by $g_{s}$ the real mapping on $X$ defined as $g_{s}(x)=g(x, s)$ for each fixed $s \in Y$.

We say that $\Gamma$ is an upper semicontinuous game if both $f$ and $g$ are upper semicontinuous. Moreover, we say that $\Gamma$ is an upper Baire game if $f_{t}=\bar{f}_{t}$ for all $t \in X$ and $g_{s}=\bar{g}_{s}$ for all $s \in Y$. Finally, we say that $\Gamma$ is a quasi-concave game if the mapping $x \mapsto f(x, y)$ is quasi-concave for each fixed $y$ and the mapping $y \mapsto g(x, y)$ is quasi-concave for each fixed $x$.

Proposition 1 Let $\Gamma$ be an upper semicontinuous and upper Baire game. Assume that $\left\{x_{n}\right\} \subset X$ is a sequence converging to some $x_{0} \in X$. Then any sequence $\left\{z_{n}\right\}$ such that $z_{n} \in R_{f, g}\left(x_{n}\right)$ admits a subsequence converging to some $z_{0} \in R_{f, g}\left(x_{0}\right)$.

Proof Fix a sequence $\left\{z_{n}\right\}$ such that $z_{n} \in R_{f, g}\left(x_{n}\right)$ for all $n \in \mathbb{N}$. Then, according to Remark 5, for every $n \in \mathbb{N}$ there exists a $y_{n} \in Y$ such that

$$
\left\{\begin{array}{l}
f\left(z_{n}, y_{n}\right) \geq f\left(t, y_{n}\right) \text { for all } t \in X \\
g\left(x_{n}, y_{n}\right) \geq g\left(x_{n}, s\right) \text { for all } s \in Y
\end{array}\right.
$$

By the Bolzano-Weierstrass theorem, we can always find a subsequence $\left\{y_{n_{k}}\right\}$ such that $\left\{y_{n_{k}}\right\}$ converges to some $y_{0} \in Y$. By the same token, there exists a subsequence $\left\{z_{n_{k_{r}}}\right\}$ such that $\left\{z_{n_{k_{r}}}\right\}$ converges to some $z_{0} \in X$. If we apply Eq. (8) to the subsequences $\left\{x_{n_{k_{r}}}\right\},\left\{y_{n_{k_{r}}}\right\}$ and $\left\{z_{n_{k_{r}}}\right\}$ and we pass to the limit as $r \rightarrow \infty$, we get

$$
\begin{cases}\lim \sup _{r \rightarrow \infty} f\left(z_{n_{k_{r}}}, y_{n_{k_{r}}}\right) \geq \limsup _{r \rightarrow \infty} f\left(t, y_{n_{k_{r}}}\right) & \text { for all } t \in X \\ \lim \sup _{r \rightarrow \infty} g\left(x_{n_{k_{r}}}, y_{n_{k_{r}}}\right) \geq \limsup _{r \rightarrow \infty} g\left(x_{n_{k_{r}}}, s\right) & \text { for all } s \in Y .\end{cases}
$$

Owing to the upper semicontinuity of $f$ and $g$, we have that $\lim _{\sup } \rightarrow \infty f\left(z_{n_{k_{r}}}, y_{n_{k_{r}}}\right) \leq$ $f\left(z_{0}, y_{0}\right)$ and $\lim \sup _{r \rightarrow \infty} g\left(x_{n_{k_{r}}}, y_{n_{k_{r}}}\right) \leq g\left(x_{0}, y_{0}\right)$, while $\limsup _{r \rightarrow \infty} f\left(t, y_{n_{k_{r}}}\right)=$ $f\left(t, y_{0}\right)$ and $\lim \sup _{r \rightarrow \infty} g\left(x_{n_{k r}}, s\right)=g\left(x_{0}, s\right)$ hold due to the upper Baire assumption on $f$ and $g$. Therefore, the previous equation goes to Eq. (6), which is exactly the desired claim.

Corollary 2 Let $\Gamma$ be an upper semicontinuous and upper Baire game. Then $R_{f, g}$ is closed-valued.

Proof Given any $x_{0} \in X$, let $\left\{z_{n}\right\} \subset R_{f, g}\left(x_{0}\right)$ be a sequence converging to some $z_{0} \in X$. The claim is shown if we prove that $z_{0} \in R_{f, g}\left(x_{0}\right)$ : this conclusion may be easily drawn by applying Proposition 1 , where $\left\{x_{n}\right\}$ is the constant sequence given by $x_{n}=x_{0}$.

Now the stage is set for the main result of this section.

Theorem 2 Assume that $\Gamma$ is an upper semicontinuous, upper Baire and quasiconcave game. Then $E(\Gamma)$ is not empty. 
Proof The assumption of upper semicontinuity of $f$ and $g$ ensures that both $R_{f}$ and $R_{g}$ are strict and so is also $R_{f, g}$. Owing to Corollary 2, we know that $R_{f, g}$ is closedvalued. According to Corollary 1, the claim is shown if Theorem 1 applies to $R_{f, g}$. We assert that $R_{f, g}$ satisfies Eq. (2). Suppose $a b$ absurdo that there exists a $x_{0} \in X$ such that

$$
\limsup _{x \downarrow x_{0}} m_{R_{f, g}}(x)<m_{R_{f, g}}\left(x_{0}\right) .
$$

Consequently, there exists a decreasing sequence $\left\{x_{n}\right\} \subset X$ converging to $x_{0}$ and a $l \in X$ such that

$$
m_{R_{f, g}}\left(x_{n}\right)<l<m_{R_{f, g}}\left(x_{0}\right) \text { for all } n \in \mathbb{N} \text {. }
$$

Set $z_{n}=m_{R_{f, g}}\left(x_{n}\right)$ : by Proposition $1, z_{n}$ being an element of $R_{f, g}\left(x_{n}\right)$, there exists a subsequence of $\left\{z_{n}\right\}$ converging to some $z_{0} \in R_{f, g}\left(x_{0}\right)$. Since obviously any subsequence of $\left\{z_{n}\right\}$ satisfies Eq. (9), we deduce that $z_{0} \leq l<m_{R_{f, g}}\left(x_{0}\right)$. At the same time, by definition of $m_{R_{f, g}}\left(x_{0}\right)$, we have that $z_{0} \geq m_{R_{f, g}}\left(x_{0}\right)$, which contradicts the previous inequality, so showing the assertion. The rest of the proof is devoted to proving that $R_{f, g}$ also verifies Eq. (1): if we follow a line of reasoning by contradiction, it is not difficult to see that, repeating the same argument as in the assertion, the existence of a $z_{0} \in R_{f, g}\left(x_{0}\right)$ such that $z_{0}>s_{R_{f, g}}\left(x_{0}\right)$ is assured. According to Remark $5, z_{0} \in R_{f, g}\left(x_{0}\right)$ is equivalent to Eq. (6): by the same token, $s_{R_{f, g}}\left(x_{0}\right) \in R_{f, g}\left(x_{0}\right)$ ensures the existence of a $y_{1} \in Y$ such that

$$
\begin{cases}f\left(s_{R_{f, g}}\left(x_{0}\right), y_{1}\right) \geq f\left(t, y_{1}\right) & \text { for all } t \in X \\ g\left(x_{0}, y_{1}\right) \geq g\left(x_{0}, s\right) & \text { for all } s \in Y\end{cases}
$$

First of all, observe that Eqs. (6) and (10) imply

$$
g\left(x_{0}, s\right) \leq g\left(x_{0}, y_{0}\right)=g\left(x_{0}, y_{1}\right) \text { for all } s \in Y \text {. }
$$

Suppose first that $y_{0}=y_{1}$ : due to the quasi-concavity of $t \mapsto f\left(t, y_{0}\right)$, we derive that every $x \in\left[s_{R_{f, g}}\left(x_{0}\right), z_{0}\right]$ belongs to $R_{f, g}\left(x_{0}\right)$, so contradicting the definition of $s_{R_{f, g}}\left(x_{0}\right)$. The last step is to prove that even the case $y_{0} \neq y_{1}$ leads to the same contradiction.

Assume, without loss of generality, that $y_{0}<y_{1}$ : we state that for every $x \in$ ]$s_{R_{f, g}}\left(x_{0}\right), z_{0}$ [ there holds a $y \in\left[y_{0}, y_{1}\right]$ such that $x \in R_{f}(y)$. Suppose ab absurdo that there exists a $\bar{x} \in] s_{R_{f, g}}\left(x_{0}\right), z_{0}\left[\operatorname{such}\right.$ that $\bar{x} \notin R_{f}(y)$ for every $y \in\left[y_{0}, y_{1}\right]$. Recall that, as a consequence of the properties of upper semicontinuity and quasi-concavity of $f, R_{f}(y)$ is a nonempty compact real interval, denoted by $\left[m_{R_{f}}(y), M_{R_{f}}(y)\right]$, for all $y \in Y$. Now, let $A=\left\{y \in\left[y_{0}, y_{1}\right]: M_{R_{f}}(y)<\bar{x}\right\}$ and $B=\left\{y \in\left[y_{0}, y_{1}\right]\right.$ : $m_{R_{f}}(y)>\bar{x}$ : it is clear that $A \cup B=\left[y_{0}, y_{1}\right]$ and $A \cap B$ is empty. Moreover, the fact that $z_{0}$ belongs to $R_{f}\left(y_{0}\right)$, combined with $z_{0}>\bar{x}$, leads to $M_{R_{f}}\left(y_{0}\right) \geq z_{0}>\bar{x}$ which trivially implies that $y_{0} \in B$. Similarly, one can see that $y_{1} \in A$, thus $\{A, B\}$ is a nontrivial partition of $\left[y_{0}, y_{1}\right]$. The statement is shown if we prove that $A$ and $B$ 
are closed subsets of $\left[y_{0}, y_{1}\right]$, in contradiction with the connectedness of $\left[y_{0}, y_{1}\right]$ : we will analyze the set $A$ only (the other case is analogous). Let $\left\{y_{n}\right\} \subset A$ be an arbitrary sequence converging to some $y^{*} \in\left[y_{0}, y_{1}\right]$ : we have to prove that $y^{*}$ belongs to $A$. Note that $f\left(M_{R_{f}}\left(y_{n}\right), y_{n}\right) \geq f\left(t, y_{n}\right)$ for every $n \in \mathbb{N}$ and for all $t \in X$, hence, repeating the same argument as in the proof of Proposition 1, it is not difficult to see that the sequence $\left\{M_{R_{f}}\left(y_{n}\right)\right\}$ admits a subsequence converging to some $z^{*} \in R_{f}\left(y^{*}\right)$. Further, $y_{n}$ being an element of $A$, we have that $M_{R_{f}}\left(y_{n}\right)<\bar{x}$, hence we get the double inequality $m_{R_{f}}\left(y^{*}\right) \leq z^{*} \leq \bar{x}$, which implies that $y^{*} \in A$, so closing the statement. If we combine the statement with Eq. (11) and the quasi-concavity of $g$, we derive again that every $x \in\left[s_{R_{f, g}}\left(x_{0}\right), z_{0}\right]$ belongs to $R_{f, g}\left(x_{0}\right)$, so definitely concluding the proof.

Before concluding with a simple example in order to show the usefulness of Theorem 2, we need to recall the notion of weak transfer quasi-continuity introduced by Nessah and Tian (2008), here reframed for the class of two-player games considered in this paper.

Definition 1 A game $\Gamma$ is said to be weakly transfer quasi-continuous if, and only if, whenever the strategy profile $\left(x_{0}, y_{0}\right)$ does not belong to $E(\Gamma)$, there exists a $\left(x_{1}, y_{1}\right) \in X \times Y$ and a neighborhood $\mathcal{V}_{0}$ of $\left(x_{0}, y_{0}\right)$ so that for every $(z, w) \in \mathcal{V}_{0}$ there holds $f\left(x_{1}, w\right)>f(z, w)$ or $g\left(z, y_{1}\right)>g(z, w)$.

Example 1 Consider the game $\Gamma$ with $X=Y=[0,1]$ and

$$
f(x, y)=\left\{\begin{array}{ll}
\frac{1}{2} y, & \text { if } 0 \leq y<\frac{1}{2} ; \\
x+y+\frac{1}{4}, & \text { otherwise, }
\end{array} \text { and } g(x, y)=f(y, x)\right.
$$

We assert that such game is not weakly transfer quasi-continuous. Indeed, consider the strategy profile $\left(x_{0}, y_{0}\right)$, where $x_{0}=y_{0}=1 / 2$ : obviously, $\left(x_{0}, y_{0}\right) \notin E(\Gamma)$ since, for instance, $f\left(x, y_{0}\right)>f\left(x_{0}, y_{0}\right)$ for every $x>x_{0}$. Then, for any $\left(x_{1}, y_{1}\right) \in X \times Y$ and any (sufficiently small) neighborhood $\mathcal{V}_{0}$ of $\left(x_{0}, y_{0}\right)$, picking $(z, w) \in \mathcal{V}_{0}$ with $z<1 / 2$ and $w<1 / 2$, we get $f\left(x_{1}, w\right)=\frac{1}{2} w=f(z, w)$ and $g\left(z, y_{1}\right)=\frac{1}{2} z=g(z, w)$, so proving the assertion. Thus, as shown in Nessah and Tian (2008), this game does not verify other forms of weakened continuity such as diagonal transfer continuity, better reply security, or weakly transfer continuity. Accordingly, most theorems concerning the existence of pure strategy Nash equilibria in games with possibly discontinuous payoffs cannot be applied [see for instance the results of Dasgupta and Maskin (1986), Baye et al. (1993), Reny (1999) and Nessah and Tian (2008)]. However, it is really an elementary task to check that such game is upper semicontinuous, upper Baire and quasi-concave, so the existence of a pure strategy Nash equilibrium is assured by Theorem 2.

As a final remark, we have to recall that Tian (2015) has fully characterized Nash equilibria in games with general action spaces and payoffs by means of a single condition, called recursive diagonal transfer continuity. However, as admitted by Tian himself, this condition is generally very hard to check: we simply point out that the weakened form of continuity of the payoff functions assumed in Theorem 2, 
combined with their quasi-concavity, constitutes a practical sufficient condition for recursive diagonal transfer continuity.

Acknowledgements We would like to thank an anonymous Reviewer for his careful reading and detailed comments that have been helpful for providing a better version of the present work.

Funding Open access funding provided by Università degli Studi di Ferrara within the CRUI-CARE Agreement.

Open Access This article is licensed under a Creative Commons Attribution 4.0 International License, which permits use, sharing, adaptation, distribution and reproduction in any medium or format, as long as you give appropriate credit to the original author(s) and the source, provide a link to the Creative Commons licence, and indicate if changes were made. The images or other third party material in this article are included in the article's Creative Commons licence, unless indicated otherwise in a credit line to the material. If material is not included in the article's Creative Commons licence and your intended use is not permitted by statutory regulation or exceeds the permitted use, you will need to obtain permission directly from the copyright holder. To view a copy of this licence, visit http://creativecommons.org/licenses/by/4.0/.

\section{References}

Amir R, De Castro L (2017) Nash equilibrium in games with quasi-monotonic best-responses. J Econ Theory 172:220-246

Aubin JP, Frankowska H (2009) Set-valued analysis. Springer, Berlin

Baye M, Tian G, Zhou J (1993) Characterizations of the existence of equilibria in games with discontinuous and non-quasiconcave payoffs. Rev Econ Stud 60:935-948

Dasgupta P, Maskin E (1986) The existence of equilibrium in discontinuous economic games, I: Theory. Rev Econ Stud 53:1-26

Kakutani S (1941) A generalization of Brouwer's fixed point theorem. Duke Math J 8:457-459

Kelley JL (2017) General topology. Courier Dover Publications, USA

Milgrom P, Roberts J (1994) Comparing equilibria. Am Econ Rev 84:441-459

Nessah R, Tian G (2008) The existence of equilibria in discontinuous and nonconvex games, Working paper

Reny PJ (1999) On the existence of pure and mixed strategy nash equilibria in discontinuous games. Econometrica 67:1029-1056

Tarski A (1955) A lattice-theoretic fixed point theorem and its applications. Pac J Math 5:285-309

Tian G (2015) On the existence of equilibria in games with arbitrary strategy spaces and preferences. J Math Econ 60:9-16

Publisher's Note Springer Nature remains neutral with regard to jurisdictional claims in published maps and institutional affiliations. 